\title{
Halifax 1940 : port de transit pour l'or européen
}

\section{Marie de Lavigne-Aubery}

Europe had been invaded and Britain stood alone to face Hitler's armies. As it was imperative that the gold reserves in Europe's central banks be protected from German greed, the gold had to be sent to North America in warships. With access to US ports limited by the Convention on maritime neutrality, the Allies chose the port of Halifax, Nova Scotia, as a transit hub for the European treasure. The gold was unloaded in the utmost secrecy and transported by rail to the Bank of Canada in Ottawa and the Federal Reserve in New York.

Le samedi 13 juillet 1940 à 8 h, Sydney Perkins regarde le vieux cuirassé britannique de 30000 tonnes, HMS Revenge qui, aidé de deux remorqueurs, vient de s'amarrer au quai numéro 6 du port d'Halifax. En attendant le déchargement du navire, le souvenir d'un matin de décembre 1917 il était chauffeur sur le Niobe de la Marine royale canadienne lui revient : «J'étais debout sur la plage avant du croiseur, lorsque je remarquai un incendie sur le pont d'un navire français à environ 800 mètres. À ce moment-là, près de la moitié d'Halifax sauta. Le transport de munitions Mont Blanc, chargé de 3000 tonnes de nitroglycérine et d'essence était entré en collision avec le vapeur norvégien Imo ... Sur le Niobe, quinze officiers et marins moururent et une centaine furent blessés. J'eu une chance étonnante; j'ai été projeté de la plage avant sur la chaîne de l'ancre, d'où je réussis à ramper à l'abri au milieu d'une pluie de fragments de métal et de débris enflammés »' Sydney Perkins sera le survivant le plus proche de l'explosion qui fera plus de 1 950 morts et 9000 blessés et transformera Halifax en une ruine fumante.

Que fait Sydney Perkins, alors représentant de la Banque du Canada, sur les quais du port d'Halifax? Un train spécial de la compagnie Canadian National Express avec douze wagons plombés attend sur le quai, protégé par des gardes armés ${ }^{2}$.

1 Alfred Draper, Opération Fish. Comment l'or de l'Europe échappa aux Nazis. 1939-1945 (Paris: Plon, 1979), 181.

2 Note sur les transports d'or à New York, 10 janvier 1940. Archives Banque de France, Paris, The Northern Mariner / Le marin du nord, XVIII, No. 2 (Spring 2018), 139-57 
Toute la zone est interdite d'accès et personne ne s'explique ce qui s'y passe. Alors que Sir Otto Niemeyer, administrateur de la Banque d'Angleterre, quelques fonctionnaires du Trésor britannique et le général Sikorski, dirigeant polonais, sont encore à bord, le déchargement de la cargaison commence. Après vérification de toutes les caisses par Perkins et leur chargement sur le train pour Ottawa, HMS Revenge fait un mouvement pour laisser la place au HMS Bonaventure, un des croiseurs les plus modernes de la Royal Navy, afin de continuer l'évacuation de la fortune de la Grande-Bretagne. Une grande partie de l'or de l'Europe transite par le port d'Halifax pour être sauvegardée et protégée dans les chambres fortes de la Réserve fédérale de New York et de la Banque du Canada à Ottawa. L'Opération Fish, coup de poker de Churchill sur la fortune britannique, fait suite à l'évacuation de l'or français ${ }^{3}$ vers une même destination : l'Amérique du Nord.

Face à la dégradation de la situation politique et militaire, une Europe envahie et en déroute laissant la Grande-Bretagne isolée face aux armées de Hitler, mettre l'or de l'Europe à l'abri des convoitises allemandes pour payer les dépenses de guerre deviendra une impérieuse nécessité. Seules les infrastructures du port d'Halifax en Nouvelle-Écosse permettent le transit de la fortune européenne. Mais au moment de la capitulation de la France et face au fardeau laissé par celle-ci, comment la Grande-Bretagne ne sera-telle pas tentée par les 254 tonnes d' « or de Vichy » convoyées sur l'Émile Bertin lorsque celui-ci arrivera sur les quais du port d'Halifax?

\section{De l'or pour la guerre : aspects politiques et économiques}

\section{Convoitises nazies sur l'or de l'Europe}

À l'aube du $1^{\text {er }}$ septembre 1939, la Wehrmacht attaque la Pologne. Plus de 1 500 avions décollent et 56 divisions envahissent le pays au moment où les armées soviétiques brisent la résistance polonaise. C'est le désastre, la « guerre éclair » ou Blitzkrieg. Le 3 septembre, la France et la Grande-Bretagne déclarent la guerre à l'Allemagne. Sur mer, les bâtiments anglais sont attaqués par des sous-marins ennemis. La Bataille de l'Atlantique commence. Et puis, tout s'arrête comme par miracle, c'est la « drôle de guerre » jusqu'à cette nuit du 8 avril 1940. Il est près de minuit, le roi de Norvège Haakon VII travaille encore dans son bureau. Les sirènes d'alerte aériennes se mettent à hurler. Les Nazis n'ont pas respecté la neutralité du pays et les navires de guerre allemands escortant des transports de troupes s'avancent vers les côtes. L'ultimatum présenté par l'ambassadeur allemand en Norvège, le Dr Curt Brauer, est rejeté. C'est la guerre. Il faut évacuer au plus vite le gouvernement et la famille royale mais aussi les réserves d'or : sans or, plus de combat. Immédiatement le Ministre des Finances Oscar Torp donne l'ordre d'évacuer 50 tonnes d'or en lingots et pièces du centre d'Oslo vers

Boîte 49, Bordereau 1060200101.

3 Une partie de cet or (environ 1490 tonnes) sera évacuée vers l'Afrique du nord. 
un lieu sûr. Pendant deux mois, l'or de la Norvège vivra une incroyable odyssée qui le conduira, grâce à un certain Fredrik Haslund, au nord du cercle arctique afin qu'il soit transféré le 26 mai à la Banque d'Angleterre, à Londres, sur le croiseur britannique HMS Enterprise. Après la Norvège, ce sont les Pays Bas, le Luxembourg, la Belgique et, enfin, le 5 juin commence l'ultime épisode de la « Bataille de France ». Avec ses « 126 divisions, la totalité de son immense armée cuirassée, forte de dix divisions de Panzer comprenant près de 3000 véhicules blindés, dont un millier au moins étaient des chars lourds $\gg^{4}$, sans compter les avions bombardiers, la Wehrmacht se lance à travers les Ardennes perçant à Sedan le 14 mai, brisant la ligne Maginot le 14 juin pour arriver à Paris jusqu'à ce que la France capitule et signe l'armistice le 22 juin 1940.

L'Allemagne nazie rêve surtout d'accéder au stock d'or de la Banque de France. L'armistice n'est pas encore signé entre l'Allemagne et la France, que deux officiers de l'armée du Reich se présentent au siège de la Banque de France, à Paris. Ils veulent s'assurer de la présence des quelques 2430 tonnes d'or protégées dans ses sous-sols et constituées des réserves métalliques de la France mais aussi des dépôts confiés par une dizaine de pays dont la Belgique et la Pologne. Capturer le Trésor de l'ennemi, n'est-ce pas la première victoire dans les conflits entre États ? Mais, il n'y a plus rien dans la « Souterraine », ce gigantesque coffre-fort de onze mille mètres carrés fermé par une tourelle en acier de 130 tonnes qui se trouve au huitième sous-sol du 20, rue Colonel le Drian à Paris. Par rapport au reste de l'Europe, les Nazis ont peu d'or. Ils ont environ 100 millions \$US en or lorsqu'ils commencent la guerre et sont dans l'impossibilité de financer leur économie de guerre avec leur propre monnaie. Récupérer l'or des nations européennes est donc capital pour l'Allemagne car peu de nations neutres acceptent la monnaie du Reich. Si Hitler a pu obtenir du pétrole de l'Union Soviétique puis de la Roumanie sans contrepartie en or, certains métaux essentiels à l'économie de guerre, comme le minerai de fer, l'étain, le tungstène, le cobalt ou certains produits manufacturés doivent être importés de pays neutres (Portugal, Espagne, Suède, Suisse, Turquie). L'Allemagne pillera plus de 580 millions $\$ U^{5} S^{5}$ dans les chambres fortes des banques centrales des pays européens envahis ${ }^{6}$, dont l'or de la Belgique (223 millions \$US), des Pays-Bas (168 millions \$US), des Autrichiens, Hongrois, Yougoslaves, Tchèques, Polonais et Luxembourgeois ${ }^{7}$. Mais, il leur en faudrait bien plus ${ }^{8}$.

4 Winston Churchill, Mémoires de Guerre, Tome 1, 1919-février 1941 (Paris: Collection Texto, Tallandier, 2015), 407.

5 Stuart Eizenstat, US and Allied Efforts to Recover and Restore Gold and Other Assets Stolen or Hidden by Germany During World War II, (Washington, DC: Department of State, May, 1999).

6 Duncan McDowall, Due Diligence: A Report on the Bank of Canada's Handling of Foreign Gold During World War II (Ottawa: Bank of Canada, 1997), http://www.bankofCanada.ca/wpcontent/uploads/2010/07/gold97-4.pdf.

7 Adam Lebor, Hitler's Secret Bankers: The Myth of Swiss Neutrality During the Holocaust (New York: Citadel Press, 1997), 237-241. Ces proportions sont une estimation des Alliés. Au cours des années sur lesquelles porte le présent rapport, le prix de l'or était fixé à 35 \$US.

8 http:/histclo.com/essay/war/ww2/eco/gold/w2e-gold.html « World War II Economics: Gold ». 
Réserves d'or en 1936

$\begin{array}{lcrr} & \begin{array}{c}\text { Or } \\ \text { tonnes }\end{array} & \begin{array}{c}\text { PIB* } \\ \text { (milliards \$US 1990) }\end{array} & \begin{array}{c}\text { Populations } \\ \text { (millions } \\ \text { d'habitants) }\end{array} \\ \text { États-Unis } & 9051 & 798 & 129 \\ \text { France } & 3768 & 178 & 42 \\ \text { Grande-Bretagne } & 1471 & 284 & 47 \\ \text { Belgique } & 503 & 41 & 8 \\ \text { Suisse } & 438 & 25 & 9 \\ \text { Pays-Bas } & 432 & 44 & 9 \\ \quad \text { *PIB ou Produit Intérieur Brut. Indicateur économique de la richesse produite par } \\ \text { an et par pays. } \\ \quad \text { Arnaud Manas, L'or de Vichy, (Paris:Vendémiaire, 2016), 23. Les réserves d'or } \\ \text { sont issues du rapport de la BRI (Banque des Règlement Internationaux) de 1936. }\end{array}$

\section{Les Alliés : les contrats d'armement et la clause américaine "Cash \& Carry»}

Les Alliés, eux aussi, ont besoin d'or pour les mêmes raisons que les Allemands: ils ont besoin d'armes. Très tôt, les Français, conscients de leur infériorité aéronautique face aux Allemands, se sachant incapables de produire assez rapidement des avions modernes, se tournent vers les États-Unis. Pour Pierre Cot, Ministre Français de l'Air, seuls les États-Unis peuvent fournir ces compléments d'armements indispensables à la guerre. C'est une petite commande française (100 Curtiss-Wright P-36 et 20 Chance Vought) de juillet 1938 qui va lancer les lignes de fabrication d'avions américains nécessaires aux Alliés 9 . Après Munich, entre février et mai 1939, 665 avions et 2533 moteurs seront commandés aux Américains pour environ 84,1 millions \$US. Cette première commande d'avions sera expédiée en France avant la déclaration de guerre. En août 1939, les Français passent une nouvelle commande de 1250 appareils $^{10}$.

Les firmes américaines demandent une avance de 20 à $50 \%$ sur le prix de vente, irrécupérable en cas d'interdiction de livraison. En effet, les États-Unis sont alors neutres et les lois de neutralité (Neutrality Acts) initiées par une volonté

9 Gérard Bossuat, Les Aides Américaines Économiques et Militaires à la France, 1938-1960. Une nouvelle image des rapports de puissance (Paris: Comité pour l'Histoire Economique et Financière de la France. Ministère de l'Economie, des Finances et de l'Industrie, 2001), Chapitre premier « Des avions américains pour la France».

10 Selon le Ministère des Finances, 2035 avions et 8805 moteurs ont été commandés de mars 1938 à novembre 1939 pour la bagatelle de 337 millions \$US. Ce furent les « commandes françaises » d'avant l'installation de la Commission d'achat franco-anglaise en décembre 1939. Après, on parlera de « commandes alliées ». Le 20 février 1940, le Conseil suprême de la Guerre franco-britannique adopte un programme commun de commandes d'avions aux États-Unis pour 1941. Le programme allié concernait l'achat de 4700 cellules et moteurs livrables d'octobre 1940 à octobre 1941, d'un montant total de 614 millions \$US. La part française était de 2200 avions. 
Commandes françaises par types d'avions (mars 1938 à septembre 1939)

\author{
Type d'avions \\ Curtiss P-36 \\ Curtiss P-40 \\ North American ET9B et NA-64 \\ Glenn-Martin 167-F \\ Douglas DB-7 et DB 7A \\ Chance Vought 156 \\ Total
}

$\begin{aligned} \text { Nombre } & \text { Remarques } \\ 750 & \text { Chasseur } \\ 100 & \text { Chasseur, évolution du P-36 } \\ 430 & \text { Entraînement, biplace } \\ 345 & \text { Bombardier } \\ 370 & \text { Bombardier moderne } \\ 40 & \text { Attaque au sol en piqué } \\ 2035 & \end{aligned}$

Source: Bossuat, 11.

d'isolationnisme et de non-interventionnisme dans les conflits étrangers, votées dans les années 30 (1935-1936 et deux en 1937) interdisent tout prêt ainsi que toute livraison d'armes par les Américains aux belligérants, quels qu'ils soient. Dans la deuxième loi de Neutralité de 1937, Roosevelt réussit à introduire pour deux ans, la clause du Cash and Carry, imaginée par Bernard Baruch afin d'aider les Alliés. Cette clause « imposait le paiement en dollars ou en or et l'obligation pour l'acheteur de transporter à ses frais et à ses risques la marchandise achetée $»^{11}$.

Mais après l'invasion de la Chine par le Japon en 1937 cette clause, mise à mal par les isolationnistes, n'est pas renouvelée. Ce n'est qu'en novembre 1939, à la suite de l'invasion de la Pologne et du déclenchement de la Seconde Guerre mondiale que Roosevelt, surmontant l'opposition des isolationnistes, réussit à refaire passer, dans le Neutrality Act du 4 novembre 1939, la clause du Cash and Carry qui met fin à l'embargo sur les armes.

Mais ce n'est pas tout : la France n'ayant pas liquidé ses dettes de la Première Guerre mondiale et le Johnson Act de 1934 interdisant tout prêt aux pays en défaut de paiement, celle-ci n'a alors aucun moyen de payer ses fournisseurs nordaméricains si ce n'est en dollars ou en or. Les paiements en or des avions et des moteurs débuteront en novembre $1939^{12}$. Il faut donc de l'or pour l'économie de guerre, non seulement pour l'Allemagne mais surtout pour la France et son alliée, la Grande-Bretagne.

11 Manas, 157 n132.

12 Bossuat, 12. « Des navires français convoyèrent l'or jusqu'au port d'Halifax au Canada. De là, des trains blindés le transportèrent vers New York. Il y avait aux États-Unis, en juin 1940, 430,6 tonnes d'or fin appartenant à la Banque de France et 15,7 tonnes appartenant au Fond de Stabilisation des Changes, ainsi que 232,4 millions \$US comme gages des commandes américaines (...). Les avions étaient à leur tour transportés à Halifax, d'où des cargos français ramenaient les appareils sous protection de la Marine nationale ». 


\begin{tabular}{|c|c|c|c|c|c|c|c|}
\hline \multicolumn{8}{|c|}{$\begin{array}{l}\text { Synthèse des achats français aux États-Unis, 1938-16 juin 1940, d'après le } \\
\text { rapport Bloch-Lainé du } 19 \text { août } 1940 \\
\text { (En millions } \$ \text { US) }\end{array}$} \\
\hline riode & $\begin{array}{l}\text { Va l e u r } \\
\text { contrats }\end{array}$ & Paiements & Avances & $\begin{array}{l}\text { Paiements } \\
\text { au profit } \\
\text { du BPC 16- } \\
25 / 6 / 40\end{array}$ & $\begin{array}{r}\text { tr a t s } \\
\text { a) }\end{array}$ & $\begin{array}{l}\text { Côuts } \\
\text { éels }\end{array}$ & $\begin{array}{l}\text { Avions } \\
\text { livr és } \\
\text { (France) }\end{array}$ \\
\hline 38-Août 39 & 90 & & & & & & \\
\hline$-31 / 12 / 39$ & 193 & 136 & 112,8 & & & & 359 \\
\hline $\begin{array}{l}\text { vrier }-\mathrm{m} \\
4015 \text { mai } 4\end{array}$ & 482 & 175,7 & 39 & & & & 672 \\
\hline & & & & & & & 980 \\
\hline al avi & 765 & 311,7 & 151,8 & 24,1 & 629,3 & 135,8 & 1377 \\
\hline tal autres (c & 249,5 & 161,5 & 57,9 & & 149,8 & 103,6 & \\
\hline nd & 1014,6 & 473,2 & 209,7 & 24,1 & 779,1 & 239,4 & \\
\hline
\end{tabular}

Note a : Ce poste correspond à des achats de machines-outils air et armements, à divers matériels d'armement, à des matières premières, des poudres et des essences, à des matériels pour la marine pour la période septembre 1939 à juin 1940.

Note b : BPC : British Purchase Council. Il s'agit de la British Purchasing Commission.)

Source: Bossuat, 20.

Un autre problème se pose : le régime de la neutralité maritime interdit l'accès aux ports américains

Au milieu du désastre, de la confusion générale qui règne en Europe, de l'exil de nombreux gouvernements et l'exode de milliers de personnes, il faut évacuer l'or européen et le convoyer sur des bateaux de guerre vers l'Amérique du Nord pour le soustraire aux convoitises nazies. Mais il est impossible, depuis la déclaration de guerre du 3 septembre, d'atteindre un port de la côte est des États-Unis à cause du régime juridique de la neutralité. «La neutralité est la politique adoptée par un État qui reste à l'écart d'une guerre affectant deux ou plusieurs États en s'abstenant d'y participer, que ce soit directement en prenant part aux hostilités ou indirectement en assistant l'un ou l'autre des belligérants ».

La neutralité, notion coutumière à l'origine, a été codifiée lors des Conférences de la Haye de 1899 et de 1907. La neutralité est donc régie par :

- la V Convention de la Haye du 18 octobre 1907;

- la XIII Convention de la Haye du 18 octobre 1907 concernant les droits et les devoirs des puissances neutres en cas de guerre maritime;

- la Convention de la Havane du 20 février 1928 concernant la neutralité maritime.

L'article 5 de la Section II de cette dernière convention stipule : «Il est défendu aux navires de guerre des belligérants de s'arrêter dans les ports ou dans les eaux d'un État neutre pendant plus de 24 heures (...). Le navire peut prolonger son 
séjour de 24 heures en cas d'avaries ou de mauvais état de la mer; mais il devra partir aussitôt que cessera la cause du retard $»^{13}$.

Dès le début de la guerre, les Alliés se trouvent pris au piège de la clause Cash and Carry et de la neutralité des États-Unis. Il faut un port en Amérique du Nord permettant l'accostage des navires devant charger le matériel de guerre et décharger l'or. De l'or et un port contre des armes. Mais aussi des banques où stocker cet or.

\section{De l'or et un port contre des armes: le port d'Halifax au Canada devient alors la porte d'entrée vers l'Amérique}

Le Canada, en guerre contre l'Allemagne depuis le 10 septembre 1940, n'est pas concerné par les « 24 heures » imposées par la Convention de la Havane de 1928, délai insuffisant pour décharger l'or et charger le matériel militaire correspondant. Dès le 3 septembre 1939, l'évacuation de l'or français est envisagée vers les ÉtatsUnis et le Canada. Dans la note de la Caisse Générale de la Banque de France en date du 10 janvier 1946, remise à Jacques Rueff ${ }^{14}$, il est noté : " La Federal Reserve Bank de New York et la Banque du Canada à Montréal ${ }^{15}$ acceptèrent de recevoir les dépôts de la Banque de France : mais on ne peut utiliser comme port de débarquement que le port d'Halifax au Canada, les États-Unis étant neutres ne pouvaient accueillir les navires de guerre français $»^{16}$.

Le port d'Halifax ou plutôt « port de la côte Est » du Canada, comme il était désigné par le gouvernement canadien dans les communiqués de guerre, sera préféré aux ports de Québec ou de Montréal pour plusieurs raisons.

- Tout d'abord, il bénéficie d'un mouillage profond pour près de 150 navires, ne gèle jamais et peut être facilement défendu contre des attaques venant de l'Atlantique. Il est vaste et profond et surtout situé à proximité des routes maritimes des grands cercles reliant l'Europe à la côte est de l'Amérique du Nord.

- Dès septembre 1939, le complexe des terminaux océaniques de la partie sud d'Halifax a été mis au service des forces armées afin de soutenir à la fois la circulation des civils et celle des militaires. Les installations du quai 2 avaient précédemment servi à la logistique militaire au cours de la Première Guerre mondiale.

13 Convention concernant la neutralité maritime, la Havane, 20 février 1928, Article 5,II.

14 Sous-gouverneur de la Banque de France en 1939 et Président de la Commission des Réparations à Paris à partir de 1945.

15 Alors que pour les Britanniques, il n'y a qu'une banque, la Banque du Canada à Ottawa, on retrouve dans les archives de la Banque de France le nom de la Royale Banque du Canada à Montréal et de la Banque du Canada à Ottawa. En fait, l'encaisse-or française ne sera convoyée qu'à la Banque du Canada à Ottawa.

16 Note de la Caisse Générale sur « l'Évacuation de l'Or de la Banque de France en 1940 » remise à Rueff le 10 janvier 1946. Archives Banque de France, Boîte 29, Bordereau 1060200101. 
- La ville est reliée au réseau de chemin de fer transcontinental. Deux compagnies de chemin de fer s'y retrouveront lors des convoyages d'or : la Canadian National Express ${ }^{17}$ et la Compagnie des chemins de fer nationaux du Canada. L'organisation des transferts de métal sera savamment organisée par les Britanniques avec l'aide des banquiers canadiens. En effet, dans une note sur les transports d'or de la Banque de France datée du 10 janvier 1940, nous pouvons lire : «À Halifax, nous avons trouvé, outre notre attaché naval : des représentants de la Banque Royale du Canada, des représentants de la Compagnie des chemins de fer nationaux du Canada, un service d'ordre assuré par la police montée canadienne, une corvée de marins canadiens chargés de transporter les caisses du quai de débarquement aux wagons spéciaux. Une fois les caisses reconnues d'après les indications extérieures et les wagons chargés, les représentants de la compagnie de chemin de fer nous ont remis un reçu de 2051 caisses dont ils avaient pris livraison $»^{18}$.

- Enfin, la Marine royale canadienne (MRC) y est présente.

Dès la déclaration de guerre, le port d'Halifax est donc prêt à soutenir une longue guerre. Halifax, figure de proue atlantique du continent américain, sera le principal point de rassemblement pour tous les convois transatlantiques. La plupart des 160 millions de tonnes de cargos, de vivres et d'armes envoyés en Angleterre transiteront par Halifax. Le port d'Halifax sera aussi le pivot central dans le transfert de l'or européen vers les chambres fortes de la Banque du Canada à Ottawa et de la Federal Reserve Bank à New York. Les plus importantes quantités d'or transitant par le port d'Halifax viendront principalement de la Grande-Bretagne et de la France. Une petite quantité viendra des autres pays neutres envahis par les Allemands dont les réserves seront passées sous protection de la Banque de France et de la Banque d'Angleterre.

\section{Des banques pour stocker l'or de l'Europe}

À Ottawa, la Banque du Canada est alors une jeune banque. Elle a ouvert ses portes le 11 mars 1935 avec à sa tête, un gouverneur de 38 ans : Graham Towers qui, tout naturellement, se tourne pour son leadership vers la Banque d'Angleterre, cette vénérable banque londonienne qui se trouve dans la City, sur Threadneedle Street et dont le gouverneur est le redoutable Montagu Norman. Towers deviendra « the most highly regarded Dominion central banker in London » ${ }^{19}$. La Banque

17 La Railways Express Company est la compagnie américaine qui fera le convoyage de l'or de Montréal à New York.

18 Archives Banque de France, Boîte 29, Bordereau 1060200101.

19 R. S. Sayers, The Bank of England 1891-1944 Vol. II (London: Cambridge University Press 
d'Angleterre y ouvrira un compte dès 1936 afin d'acheter et de stocker de l'or canadien (9 millions $\$$ d'or canadien de la Monnaie royale canadienne) puis y déposera les premières réserves d'or outre-mer pour les achats de matériel de guerre aux États-Unis. Le 31 mars 1938, Towers recevra un télégramme personnel et secret de Siepmann, le conseiller spécial de Norman : "please consider and reply by cable on Monday if possible what would be the attitude of the Bank of Canada if the Bank of England were to propose to accumulate gold in Ottawa by purchase, transfer and shipment from others centers, up to an amount of $\$ 250,000,000$ or more..."20.

Le 22 mars 1939, c'est au tour de la Banque Nationale de Belgique de demander l'aide de la Banque du Canada par l'intermédiaire de la Banque d'Angleterre. Ils pensent y placer 25 à 50 millions \$CAN d'or fin, puis c'est le tour de la Banque Nationale de Suisse, toujours par l'intermédiaire de la Banque d'Angleterre ${ }^{21}$. En effet, alors que le bruit des bottes se fait entendre en Europe, la Banque d'Angleterre conseillera aux banquiers des autres nations européennes de prendre la direction d'Ottawa et la phrase "whenever we were asked to introduce one of our Continental friends to the Bank of Canada" 22 apparaîtra régulièrement dans sa correspondance avec Ottawa. La France, sans attendre l'accord du Canada, envoie sa première cargaison d'or, 403516 onces d'or fin en avril 1939, alors qu'elle vient d'ouvrir son compte « sous dossier ». À la fin de 1939, elle a 17531 lingots, presqu'autant que la Banque d'Angleterre (environ 520 millions $\$ \mathrm{CAN}^{23}$ ).

Mais, c'est après le Blitzkrieg allemand du printemps 1940 que la pression sur l'or sera la plus importante. L'invasion allemande des pays du nord de l'Europe obligera les gouvernements européens à chercher asile pour sauvegarder l'intégralité de leurs réserves en or alors que les précédents convois ne concernaient que les « règlements » en or des commandes de matériel de guerre. Mais avant d'arriver sur les quais du port d'Halifax et être chargé dans les wagons blindés des trains de la Canadian Railways Company ou du Canadian National Express pour atteindre les centres financiers de New York, Ottawa et Montréal, l'or devait traverser l'Atlantique Nord, l'océan le plus dangereux du monde à cette époque.

\section{La logistique de l'évacuation de l'or européen et la bataille de l'Atlantique}

Dès le 3 septembre 1939, l'Atlantique est le centre d'une guerre navale sans merci. Contrôler l'océan Atlantique est aussi vital pour l'Allemagne que pour les Alliés. L'Allemagne décide, pour asphyxier les Alliés, de couper toutes les communications maritimes avec l'outre-mer, d'empêcher tout approvisionnement,

1976), 515.

20 McDowall, 9-10.

21 Courrier de Bolton à Osborne 13 avril 1939, 16-3/1, cité dans McDowall. John Osborne était conseiller du Gouverneur de la Banque d'Angleterre et Sir George Lewis French Bolton a servi comme directeur de la Banque d'Angleterre.

22 Voir par exemple la correspondance de Towers et Norman, 14 avril 1939, BOC file A18-17.

23 McDowall, 12. 
d'entraver le transport du matériel militaire comme des matières premières et, enfin, de paralyser l'économie britannique. Car, dès la capitulation de la France, l'Allemagne veut le dernier bastion de l'Europe combattante : la Grande-Bretagne. Le torpillage de l'Athenia par l'U-30 le matin du 3 septembre 1939 sonne désormais le début de l'entrée en guerre de l'arme sous-marine en tant que principale force allemande.

Avant la fin de l'année 1939, les Alliés avaient déjà perdu 750000 tonnes de navires marchands dont 60000 furent coulées par les bâtiments de ligne allemands, les Raiders, 255000 par les mines magnétiques de la Luftwaffe et 460 000 par les sous-marins. Mais, les sous-marins allemands, les U-boots et leurs torpilles, les Raiders de surface ou cuirassés de poche extrêmement performants ${ }^{24}$ comme le Graf Spee et le Deutschland, ne sont pas les seuls à attaquer la marine marchande. Les croiseurs allemands armés de canons de huit pouces ${ }^{25}$ bien plus modernes que les autres les mines magnétiques et la guerre de course entravent la navigation dans le nord de l'Atlantique déjà extrêmement dangereux pour des raisons météorologiques. Les hommes ne meurent pas uniquement des suites d'une attaque de l'ennemi, ils meurent aussi à cause des redoutables tempêtes, des brouillards, du froid, des icebergs... qui provoquent des accidents.

La Grande-Bretagne doit coûte que coûte maintenir ouverte cette ligne de communication qu'est l'Atlantique du Nord, organiser et surtout protéger les convois transatlantiques partant de Liverpool et de la Tamise et ceux qui reviennent de Halifax, Gibraltar et Freetown. Mais, pendant les deux premières années de la guerre, les convois britanniques seront mal escortés ${ }^{26}$ et peu protégés. Trop peu de navires, mal équipés, mal armés avec des équipages inexpérimentés. Pas de chasseurs en service dans le commandement de l'aviation côtière de la Royal Air Force et les radars peu nombreux sur les navires. La coopération entre la Royal Navy et la RAF est mauvaise. Les navires se retrouveront souvent seuls ou à la traîne et se feront torpiller. Comment ces tonnes d'or vont-elles parvenir à traverser un Atlantique déchaîné infesté de sous-marins ennemis?

\section{La Bataille de l'or : l'encaisse-or de la France transite vers les quais du port d'Halifax}

En France, la mise à l'abri de l'or est préparée par une instruction générale émise par la Direction de la Sureté Générale du Ministère de l'Intérieur en date du 4 février 1930 et une décision du 29 janvier 1932 concernant un plan de concentration des encaisses-or dans des zones à l'abri des risques d'invasion étrangère et l'envoi en province de 20 milliards (francs) d'or en lingots ${ }^{27}$. Le $1^{\text {er }}$ septembre 1933 , les

24 Churchill, 315.

25 Ibid.

26 La Royal Navy est toujours maîtresse des « 7 mers » avec ses 500000 tonnes, mais elle est vieille, sa flotte datant de la Première Guerre mondiale. Bank of Canada archive, Ottawa.

27 Didier Bruneel, Les Secrets de l'or (Paris:Le Cherche-Midi, 2012), 111 «L'or réparti dans 51 succursales représentait l'essentiel des moyens de paiements dont la France en guerre disposait pour 
réserves d'or de la France sont à leur maximum : 5083 tonnes. À la fin de l'année 1935, il ne reste que 3918 tonnes. En 1937, 2443 tonnes. En 1938, après les accords de Munich, les plans pour l'évacuation de l'or sont affinés ${ }^{28}$. En mai 1940, les réserves d'or ne sont plus que de 2430 tonnes.

\section{Encaisse-or française}

$\begin{array}{ll}1933 & 5081 \text { tonnes } \\ 1935 & 3918 \text { tonnes } \\ 1937 & 2443 \text { tonnes } \\ 1940 & 2238 \text { tonnes* }\end{array}$

*Didier Bruneel, Les Secrets de l'or, (Paris:Le Cherche-Midi, 2012), 109.

Dès la déclaration de guerre du 3 septembre 1939, le transfert des réserves d'or commence. Les mois de répit autorisés par la « drôle de guerre » pendant laquelle rien ne se passe, permettent à la marine française, dans un premier temps, de convoyer entre septembre 1939 et avril 1940, sous la menace des U-boot allemands, plusieurs centaines de tonnes d'or vers Halifax. Le 14 novembre 1939, un premier convoi de 100 tonnes d'or, dont 275 caisses chargées à bord des croiseurs Marseillaise et Jean de Vienne et 1500 caisses de « macaroni » sur le cuirassé Lorraine, quitte Toulon précédé du contre-torpilleur Aigle. Après avoir torpillé deux U-boot ennemis, il accoste à Halifax avec sa cargaison d'or. Le 13 décembre 1939, le deuxième convoi quitte Brest pour Halifax avec 100 tonnes $^{29}$ d'or chargées sur le cuirassé Dunkerque, convoyées par Billetterie, contrôleur général et Katow, caissier de la Banque de France. Concernant ces deux premiers convois, on peut lire dans une «note relative aux transports d'or » de la Banque de France, en provenance de New York et datée du 11 janvier 1940 « à mon arrivée à Halifax le $1^{\text {er }}$ décembre 1939 , je trouvai à ma grande surprise, un train spécial avec une garde de police à notre disposition immédiate pour le transport de notre chargement à Ottawa. Le représentant à Halifax de la Banque du Canada avait reçu des ordres de son siège, alerté 3 jours auparavant par le Ministre de France à Ottawa, et la Banque du Canada avait spontanément mis à notre disposition l'organisation prévue pour les convois de la Banque d'Angleterre. Sur cette assurance, j'utilisais les services de la Compagnie des chemins de fer nationaux du Canada d'Halifax à Montréal et ceux de la National Railways Express (compagnie américaine) de Montréal à New York ${ }^{30}$. Le troisième convoi vers Halifax quitte Toulon le 12 mars 1940 avec 147 tonnes d'une valeur de 7 milliards de francs à bord du croiseur Algérie et du cuirassé Bretagne, convoyées par Paul Gargam et Poissonnier. Au total, 347 tonnes d'or viennent de quitter la France pour Halifax.

se procurer outre-Atlantique les armes - les avions surtout - qui lui faisaient défaut ».

28 Didier Bruneel, La Banque de France et la Seconde Guerre Mondiale(Paris: Banque de France/ Eurosystème, 2002), 11.

29 Note sur l'évacuation de l'or de la Banque de France en 1940 du 10 janvier 1946 remise au Gouverneur Rueff : « pour diviser les risques, chaque convoi ne transporta au début que 5 milliards de francs (100 tonnes d'or fin), puis, dès la mi-mai, les évacuations s'accélérèrent et l'on accepta un risque plus grand », Archives Banque de France, Boîte 29, Bordereau 1060200101.

30 Archives Banque de France, Boîte 29, Bordereau 1060200101. 
Mais la « drôle de guerre » se termine. Les événements s'accélèrent et prennent mauvaise tournure avec l'offensive allemande du 10 mai 1940. Le 14 mai 1940, le Ministre des Finances, Lucien Lamoureux, s'inquiète de la situation militaire et demande sous sa seule responsabilité à Bouthillier, son secrétaire général et Fournier, le gouverneur de la Banque de France, de préparer l'évacuation de l'intégralité des réserves d'or vers sa destination finale : l'Amérique du Nord $^{31}$. Il faut transporter l'or, dispersé dans les 51 dépôts, vers les ports de Toulon, du Verdon et de Brest au milieu d'une désorganisation totale : wagons vétustes, camions manquants, un véritable chaos sur les routes embouteillées par d'immenses colonnes de civils et de réfugiés fuyant l'ennemi. Tandis que les zones de combat se rapprochent de Brest, point le plus important de l'opération de sauvetage.

Un premier convoi quittera Toulon le 17 mai 1940, 194 tonnes sont chargées sur le porte-avions Béarn pour la Federal Reserve Bank via Halifax. Le deuxième convoi quitte Brest le 21 mai 1940 avec 224 tonnes d'or chargées sur les croiseurs Jeanne d'Arc et Émile Bertin qui rejoignent au large des Canaries le porte-avions

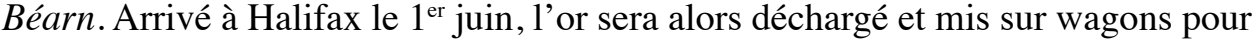
être livré à la Federal Reserve Bank à New York. Le troisième convoi concerne le paquebot Pasteur de la Compagnie Sud-Atlantique qui, transformé en croiseur auxiliaire, rejoint Brest et appareille pour Halifax le 2 juin 1940 avec $213^{32}$ tonnes d'or (3 700 sacs) représentant 10 milliards de francs. Cette cargaison sera déchargée vers la Banque du Canada à Ottawa, pour y être conservée sous le dossier Banque de France, convoyée par la Compagnie des chemins de fer nationaux du Canada ${ }^{33}$. Cet or doit financer l'achat de canons de $75 \mathrm{~mm}$ qui seront chargés à New York le 13 juin.

Mais, le 2 juin 1940, alors que la Wehrmacht envahit la France et que près de mille tonnes d'or n'ont pu être embarquées, le porte-avions Béarn attend, à quai à Halifax, le chargement d'une commande pour la France de 96 avions dont 48 bombardiers Curtis-Wright SBC-4, 23 avions de chasse et 25 de liaison. Quant à l'Émile Bertin, croiseur léger de 117 mètres, il revient d'Halifax et arrive à Brest le 9 juin. L'Émile Bertin repart de Brest avec 254 tonnes d'or (12 milliards de francs) convoyées par Katow, composées de 3986 sacs et sacoches remplis de pièces d'or et 796 caisses de lingots qui doivent être déchargés à Halifax et transportés par train spécial de la Compagnie des chemins de fer nationaux du Canada ${ }^{34}$ à la Banque Centrale du Canada à Ottawa.

31 Note sur l'évacuation de l'or de la Banque de France en 1940 du 10 janvier 1946 remise au Gouverneur Rueff : «La guerre déclarée, le 3 septembre 1939, l'évacuation sur les États-Unis et le Canada fut envisagée ». Du même jour, note de M. Rousseau lors de la visite de M. Rueff au Sénat : «M. Paul Reynaud écrit actuellement ses Mémoires et aurait l'intention de dire que l'or de la Banque de France a été dirigé sur l'Afrique Occidentale Française en violation de ses ordres, qui étaient de tout envoyer en Amérique».

32 Bruneel, Les Secrets de l'or, 114.

33 Lettre de J. Poissonnier au Gouverneur de la Banque de France, 15 juillet 1940, Archives Banque de France, Boîte 368, Bordereau 1280200801.

34 Dans « Relation des incidents qui se sont produits au Canada les 18,19, 20 et 21 juin 1940 » 


\section{Situation géographique de l'encaisse-or de la Banque de France} Londres

Ottawa

New York

Martinique

Kayes

Casablanca/Alger
Juin 1940

Juin 1944*

226,88 tonnes

215,32 tonnes

430,64 tonnes

254,15 tonnes

652,36 tonnes
226,88 tonnes

213,32 tonnes

430,64 tonnes

254,15 tonnes

334,82 tonnes

317,22 tonnes

*Juin 1940 et 1944 : Documents manuscrits de la Banque de France « Encaisse-or de la Banque de France au 30 juin 1944-Soir », Boîte 23, Bordereau 1280199901.

\section{L'Opération Fish et l'évacuation de l'or et des valeurs britanniques négociables}

Quant à Churchill, il s'inquiète et pense à la sombre « éventualité » d'une possible capitulation de la Grande-Bretagne après celle de la France. Aussi vat-il faire évacuer secrètement presque toute la fortune de la Grande-Bretagne vers le port d'Halifax. Non seulement l'or mais toutes les valeurs négociables sans même en informer les propriétaires. Le 21 mai 1940, Sir R.N.V. Hopkins, deuxième secrétaire au Trésor, adressera un mémorandum ultra secret à Churchill et au Chancelier de l'Échiquier, Sir Kingsley Wood, donnant la composition des réserves d'or du pays : "Nous avons 80 millions de livres d'or à Ottawa, 25 en Afrique du Sud, 6 aux Indes, 40 en transit. Nous en avons 280 millions à Londres, et l'on me dit que la Banque détient 200 millions appartenant à des clients tels que la Belgique, la Hollande et d'autres banques nationales. À 300000 livres la tonne, ces 480 millions de livres d'or représentent environ 1600 tonnes (...) $\gg^{35}$ :

Une loi présentée au Parlement le 22 mai 1940 par MM. Chamberlain et Attlee, donnant au gouvernement une autorité « pratiquement illimitée sur la vie, la liberté et la propriété de tous les sujets de Sa Majesté en Grande-Bretagne ${ }^{36}$, considérée par le Daily Telegraph comme la «mesure constitutionnelle la plus radicale jamais présentée à la Chambre des communes », permettra au gouvernement de disposer non seulement des biens de la Nation mais des valeurs étrangères détenues par des particuliers.

Le 22 juin à 18 h, des wagons plombés et gardés quittent Londres vers Glasgow. C'est le convoi « Big One ». Le croiseur HMS Emerald est chargé de 2229 caisses de lingots valant 30 millions de livres et de quelques 500 boîtes contenant 200 millions de livres de titres (montant nominal). Alfred Draper raconte que 1'or était arrimé dans des soutes à munitions et les boîtes de titres dispersées dans

concernant l'Émile Bertin, on cite la Canadian National Express, Archives Banque de France, Boîte 269, Bordereau 12802200801.

35 Draper, 123-124.

36 Churchill, 492. 


Or nous appartenant
Détenu par la Banque
d'Angleterre pour ses clients
Détenu par d'autres banques
pour des clients étrangers
France
Belgique
Norvège
Pays-Bas
India Office
Tchécoslovaquie
Suisse
Argentine
Total
* Draper, 128.
\# Manas, 92.

280 millions de livres*

270 millions de livres

8 millions de livres

70 millions de livres

85 millions de livres

10 millions de livres

25 millions de livres

5 millions de livres

8 millions de livres

20 millions de livres

5 millions de livres

786 millions de livres
1037 tonnes d'or fin ${ }^{\#}$

1000 tonnes d'or fin

30 tonnes d'or fin

258 tonnes d'or fin

315 tonnes d'or fin

37 tonnes d'or fin

93 tonnes d'or fin

19 tonnes d'or fin

30 tonnes d'or fin

74 tonnes d'or fin

19 tonnes d'or fin

2912 tonnes d'or fin

tous les coins du navire. Le 23 juin, le commandant Francis Flynn donne l'ordre de larguer les amarres. À la pointe de l'Irlande, la tempête sévit pendant trois

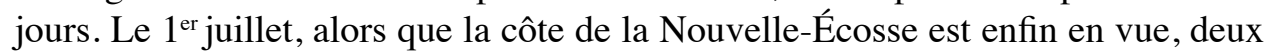
destroyers canadiens viennent à sa rencontre pour l'escorter jusqu'au port. À 7 h 35 le navire est amarré le long d'un quai. Un train spécial avec une douzaine de wagons plombés attend, entouré de fonctionnaires de la Banque du Canada et de surveillants armés de la compagnie Canadian National Express. L'or est mis à terre, compté et chargé dans les wagons. À $19 \mathrm{~h}$, le train démarre et file vers Montréal. Il est dédoublé sur une voie de garage de la gare de Bonaventure de Montréal : l'or part pour Ottawa afin d'être gardé dans les caves de la Banque du Canada alors que les titres sont conduits au centre de Montréal, dans l'immeuble de la compagnie d'assurances Sun Life. Un immeuble de 24 étages, le plus grand immeuble commercial du Commonwealth. Le sous-sol comportait trois niveaux dont le plus bas, à 15 mètres au-dessous du niveau de la rue, allait devenir « le Dépôt des valeurs du Royaume-Uni ».

Le 8 juillet 1940, le cuirassé de 30000 tonnes Revenge et le croiseur Bonaventure quittent la Clyde et rejoignent trois ex-paquebots de luxe : le Monarch of Bermuda, Batory et Sobiesky (les deux derniers appartenant à la Pologne libre). Ils transportent plus de 450 millions de livres. Après de sérieux incidents, le Revenge, le Monarch of Bermuda et le Sobiesky arrivent à Halifax. À 8 h, le Revenge s'amarre au quai numéro 6 alors que le train spécial attend. Puis c'est le tour du Bonaventure, puis du Monarch of Bermuda et enfin des deux bâtiments polonais. Cinq trains spéciaux sont nécessaires pour transporter l'intégralité du chargement surveillé par 300 hommes de la compagnie des chemins de fer. On sépare de nouveau les wagons : pour Montréal (les titres) et Ottawa (l'or).

Plus tard, d'autres navires arrivent au Canada, navires de guerre ou bâtiments de 
commerce avec un peu d'or. L'Emerald évacue encore 10 millions de livres et le Revenge 14,5 millions. Le HMS Esperance Bay, qui convoie 10 millions de livres d'or, est attaqué par une formation de Heinkel 111 et de Junkers 86 avec bombes et mitraillages. Une bombe touche le navire et tue sept hommes. Malgré les dégâts, le navire rallie Plymouth avec sa cargaison intacte. Pas un lingot ne fut perdu dans cette incroyable aventure.

\section{Les désillusions britanniques après la capitulation de la France}

\section{L'inquiétude de Churchill}

1940, la France et la Grande-Bretagne vivent les moments les plus difficiles. De dramatiques changements se produiront dans les relations entre ces deux pays qui d'alliés deviendront ennemis. Au début de la guerre, les relations sont harmonieuses. En novembre 1939, les Alliés mettent en place un Conseil Suprême de la Guerre (Supreme War Council) pour coordonner leurs stratégies. Puis un Comité d'Achat anglo-français (Anglo-French Purchasing Committee) et un Comité anglo-français de coordination (Anglo-French Coordinating Committee) dirigé par Jean Monet. Ils signent un accord financier (décembre 1939) ainsi qu'un accord commercial (16 février 1940) et un accord industriel (7 mars 1940). À la signature de l'Armistice, tous ces accords de coopération seront caducs, laissant la Grande-Bretagne seule face à l'immense charge de poursuivre la guerre.

1/ Selon la Déclaration franco-britannique du 28 mars 1940 signée lors de la réunion du $6^{\mathrm{e}}$ Conseil Suprême Interallié à Londres entre le premier Ministre Neville Chamberlain et le Président du Conseil Paul Reynaud, il ne pouvait y avoir de paix séparée : «Le gouvernement de la République française et le gouvernement du Royaume-Uni de Grande-Bretagne et d'Irlande du Nord s'engagent mutuellement à ne négocier ni conclure d'armistice ou de traité de paix durant la présente guerre, si ce n'est de leur commun accord (...) ». Au mois de juin 1940, Paul Reynaud, dans l'hypothèse d'un armistice, demandera au gouvernement britannique de libérer la France de cet engagement. C'est le refus britannique qui provoquera la démission du Gouvernement Reynaud et la nomination du Maréchal Pétain qui, lui, estimera ne pas être tenu par cette Déclaration.

2/ D'autre part, l'accord financier franco-britannique en date du 12 décembre 1939, signé par Sir J. Simon, Chancelier de l'Échiquier, et Paul Reynaud, alors Ministre des Finances, prônait non seulement une coopération monétaire et économique entre les deux pays mais aussi le partage des dépenses de guerre tant en dollars qu'en or : $60 \%$ pour les britanniques, $40 \%$ pour la France.

3/ Le 17 juin 1940, par décret, le Président Roosevelt bloque tous les avoirs de la France. Pour la Banque de France : «La mesure est justifiée par le désir de protéger ces biens contre une saisie éventuelle par les Allemands $»^{37}$.

4/ Ce même jour, le 17 juin 1940, le Général Weygand donne son accord

37 «Blocage des avoirs français », Archives Banque de France, Boîte 368, Bordereau 1280200801. 
pour que tous les contrats d'achats passés par la mission française aux ÉtatsUnis soient transmis aux Anglais qui en prendront livraison et en assureront la charge. Les avances sur contrats devront être remboursées par les Anglais au fur et à mesure des livraisons (plusieurs dizaines de millions de dollars) et leurs montants portés au compte du Trésor français à la Banque du Canada à Ottawa ${ }^{38}$. Il s'agit principalement des commandes d'avions. En effet, les contrats français d'armements commandés aux entreprises des États-Unis continuaient à courir, gagés par les sommes gigantesques transférées aux États-Unis. On parle de quelques 4600 avions transférés aux Britanniques sur une commande française d'environ 5954 avions et de 887 contrats d'une valeur de 801,2 millions \$US ${ }^{39}$.

5/ Face à ce fardeau financier qui désormais ne pèse plus que sur elle, la Grande-Bretagne se trouve dans une situation financière délicate. Non seulement il faut payer les commandes d'armements aux Américains, mais il faut aussi rembourser les avances payées par les Français (environ 152 millions \$US selon Gérard Bossuat). Churchill écrit dans ses Mémoires : «La Grande-Bretagne était entrée en guerre avec environ 4500 millions de dollars, en devises, en or ou en investissements aux États-Unis susceptibles d'être convertis en dollars ${ }^{40}$, ajoutant : «La reprise des contrats français en juin avait presque doublé le volume de nos dépenses à régler par clearing. En outre nous passions un peu partout de nouvelles commandes d'avions, de chars et de navires marchands..$^{41}$ (...) Jusqu'en novembre 1940, nous avions payé pour tous les biens reçus, 335 millions de dollars d'actions américaines, réquisitionnées à des particuliers britanniques en échange de livres sterling (...). Nous avions déboursé plus de 4500 millions de dollars en espèces. Il ne nous restait plus que 2000 millions de dollars, la plus grande partie sous forme d'investissements dont beaucoup n'étaient pas immédiatement réalisables. À l'évidence, nous ne pouvions poursuivre plus longtemps dans cette voie. Même en nous défaisant de tout notre or et de tous nos avoirs étrangers, nous n'aurions pas pu payer la moitié de ce que nous avions commandé, et l'extension prise par la guerre nous obligeait à en avoir dix fois plus $»^{42}$. En effet, très inquiet par l'évolution de la situation, Churchill écrira à Roosevelt : « Nous continuons à payer en dollars aussi longtemps que possible, mais j'aimerais être bien certain que, lorsque nous ne pourrons plus, vous nous livrerez la marchandise quand même ${ }^{43}$. Avant le vote de la loi américaine sur le Lend-Lease, ou prêt-bail ${ }^{44}$, la situation financière de la

38 "Transfert aux Anglais des contrats d'achats passés en Amérique par la mission française », Archives de la Banque de France, Boîte 368, Bordereau 1280200801.

39 B 47609, Rapport d'Hervé Alphan, 19 septembre 1940, n¹5480. Un rapport Bloch-Lainé du 19 août 1940 adressé à Vichy parle d'un transfert de contrats d'une valeur de 779 millions \$US. Cité par Bossuat, 19.

40 Churchill, 619.

41 Ibid., 621.

42 Ibid., 622.

43 Draper, 21.

44 Ce n'est que le 11 mars 1941 que Roosevelt fera voter la loi «Lend-Lease », c'est-à-dire "prêt à bail » permettant la cession gratuite de matériels de guerre au Royaume-Uni et plus tard aux autres 
Grande-Bretagne est alarmante.

L'or devient alors un objectif stratégique pour Churchill ${ }^{45}$ qui tente de contrôler celui de la Banque de France déjà déposé à Londres (258 tonnes d'or sous dossier) et convoyé secrètement par les Britanniques lors de l'Opération Fish à la « Shadow Bank of England » d'Ottawa ainsi, que celui envoyé par la France directement à Ottawa (345 tonnes d'or sous dossier). Mais, il va surtout tenter de s'emparer des stocks d'or de la France en cours de transit à Halifax ${ }^{46}$, lors du passage de l'Émile Bertin.

Dès le dimanche 23 juin, on peut lire dans le procès-verbal de la séance du Cabinet de Guerre qui se tient à Londres, que le Béarn transportant des avions en provenance des États-Unis et l'Émile Bertin chargé de 60 millions $£$ d'or ont été détournés vers la Martinique. Le Premier Ministre britannique est conscient de l'importance de la détention de ces deux bâtiments, en particulier de celui qui transporte l'or ${ }^{47}$. Un message secret est alors envoyé au Premier Lord de l'Amirauté l'informant qu'en cas de nécessité, l'or serait saisi par la force : «Cet or appartient au gouvernement français; mais il résulte des clauses de l'armistice que le gouvernement actuel n'est qu'un gouvernement fantoche et que s'il conserve l'or il est hors de doute qu'il devra le livrer aux Allemands. Ce que nous voulons éviter $»^{48}$.

\section{Incidents sur les quais d'Halifax : l'Émile Bertin et ses 254 tonnes d'or}

Mais, revenons au port d'Halifax. Après quelques jours de traversée de 1'Atlantique sans incident, l'Émile Bertin accoste ${ }^{49}$ à Halifax le 18 juin sans se douter que la France vient de capituler. L'Émile Bertin reçoit alors l'ordre de ne pas décharger son or. Le Commandant Battet doit attendre de nouvelles instructions et les représentants de la Banque du Canada comme ceux de la Canadian National Express (CNE) sont avisés que le déchargement est différé. Un deuxième télégramme arrive demandant à Battet de rallier Fort-de-France en Martinique. Les relations entre les officiers français et leurs interlocuteurs anglo-canadiens ne sont plus les mêmes. Depuis quelques heures, les Alliés anglais sont devenus

alliés des États-Unis.

45 Manas, 92. Réserves de la Banque d'Angleterre fin mai $1940: 2912$ tonnes d'or fin dont 875 appartenant aux banques étrangères.

46 Puis à Dakar lors de l'Opération Menace en septembre 1940 avec l'accord du Général De Gaulle.

$47 \mathrm{CAB} / 65 / 7 / 72,177^{\text {th }}$ Conclusions (40), The National Archives, London: "We could announce that we could keep the gold in trust for the French Empire, but that they must fight for it. (...) The Secretary of State Foreign Affairs thought there would be no difficulty at all in justifying our seizure of the gold. We could base our action on the evident desire of the French Colonies to continue the struggle and the fact that Germany had demanded the surrender of all the French gold."

48 Cité dans les Cahiers Anecdotiques de la Banque de France, n 27, Banque de France, Eurosystème, 79.

49 Croiseur Émile Bertin. «Relations des incidents qui se sont produits au Canada les 18, 19, 20 et 21 juin 1940 », Archives Banque de France, Boîte 269, Bordereau 12802200801 et « Extrait du Journal de Route de M. Poissonnier », Archives Banque de France, Boîte 269, Bordereau 12802200801. 
les ennemis de la France. Les Anglo-Canadiens ${ }^{50}$, inquiets de la destinée de l'or, tentent de retenir l'Émile Bertin.

Le 19 juin, Battet reçoit la confirmation des ordres par l'intermédiaire de l'attaché naval français à Washington, le Commandant Benech. Mais, alors que Battet demande les facilités habituelles pour sortir du port (remorqueurs, corvées, ouverture de la passe), le commandant des autorités navales d'Halifax demande le déchargement de la cargaison. M. Staden, le Directeur de la Banque du Canada, s'oppose au départ de l'Émile Bertin au motif qu'il est mandataire unique de la France pour recevoir l'or et qu'il ne peut le laisser partir que sur l'ordre du Gouverneur. Il ajoute : «Le Gouvernement canadien a droit de saisir tout or se trouvant sur son territoire ». Les représentants de la Banque de France, convoyeurs de l'or, rétorquent que la Banque de France n'a pris aucun engagement auprès de la Banque du Canada et n'a aucune obligation de lui remettre son or. « Elle reste libre d'en disposer à son gré et de l'envoyer où bon lui semble. L'or étant sur un bateau de guerre français peut être considéré comme étant toujours sur le sol français $»^{51}$.

Le Ministre de France ${ }^{52}$ à Ottawa, M. Ristelhueber, informé de la situation, demande au Premier Ministre canadien d'autoriser le départ de l'Émile Bertin. Ce même jour, à 21 h, le Premier Ministre ne s'y oppose pas. Mais le 20 juin, aucun ordre n'est reçu et les autorités du port maintiennent leur opposition au départ du bateau. Dans l'après-midi, l'amiral britannique informe que l'Émile Bertin pourra partir le lendemain. Mais le 21 juin, nouveau contrordre, le bateau doit rester à Halifax. L'ambassadeur de France à Washington demande à nouveau l'autorisation de quitter Halifax pour Fort-de-France mais donne 1' « ordre formel d'éviter tout incident ». À $17 \mathrm{~h}$, le Chef d'état-major du Commodore canadien informe Battet qu'il n'y a plus d'opposition à son départ et que « les remorqueurs et corvées seront fournis comme d'habitude ». La sortie de l'Émile Bertin se fait sans incident le 21 juin à 18 h et le croiseur rejoint Fort-de-France où l'or est déchargé et stocké au Fort Desaix. Pendant ce temps, deux autres navires, le Béarn et ses avions « bombardiers » qui croisaient au milieu de l'Atlantique et le croiseur Jeanne d'Arc reçoivent l'ordre de regagner immédiatement Fort-de-France. L'Amiral

50 Il y avait à la Banque d'Ottawa : 213 tonnes d'or de la Banque de France transférées sur le Pasteur plus les 258 tonnes d'or transférées secrètement par la Grande-Bretagne lors de l'Opération Fish dans le cadre de ce qu'on appellera «la shadow bank of England » : 471 tonnes d'or français sont donc présentes au Canada. Selon le Trading with Ennemy Act, la France est, le 10 juillet 1940, devenue pour la Grande-Bretagne un « ennemi technique ». Celle-ci demandera au Canada de se rallier à sa position, car la Grande-Bretagne a pris le contrôle des avoirs français. Le Canada s'y ralliera le 31 juillet 1940 et mettra les avoirs français sous contrôle du Custodian of enemy property. Le lendemain, cette décision est abandonnée. Le 25 août, Churchill demande la conversion de l'or français en livres sterling car il en a besoin pour payer les commandes d'avions. Pendant plusieurs mois, le Premier Ministre canadien Mackenzie King résistera pour des raisons tant politiques qu'économiques.

51 Croiseur Émile Bertin. « Relations des incidents qui se sont produits au Canada les 18, 19, 20 et 21 juin $1940 »$, Archives Banque de France, Boîte 269, Bordereau 12802200801.

52 René Ristelhueber, diplomate français, envoyé extraordinaire et ministre plénipotentiaire nommé le 6 mars 1940, arrivera au Canada le 30 mai 1940 et y demeurera jusqu'en septembre 1942. 
Robert, Commandant en Chef de la Force Navale de l'Atlantique de l'Ouest et représentant de «Vichy » pour les îles françaises de la Caraïbe et la Guyane, est basé en Martinique. Ces 254 tonnes d'or fin resteront intactes et inutilisées dans les voûtes du Fort Desaix ${ }^{53}$ jusqu'en mars 1946.

\section{Conclusion}

La contribution du Canada à l'effort de guerre fut considérable. Plus d'un million de Canadiens servirent dans les forces armées, 42000 d'entre eux furent tués et 54000 blessés. Le Canada dépensa plus de 18 milliards \$ pour la guerre, apporta une aide diplomatique et financière aux Alliés et mit à la disposition des nations européennes envahies par l'Allemagne ses ports et ses institutions financières. La Banque du Canada servit de chambre forte à une partie des réserves d'or des banques centrales. Plus de 2586 tonnes $^{54}$ d'or y furent sauvegardées. Une autre partie de ces réserves transita par Halifax pour être acheminée à la Federal Reserve Bank américaine à New York. D'autres navires chargés d'or, en provenance des ports de l'Empire, traverseront les eaux hostiles de la route transpacifique pour Vancouver en Colombie-Britannique. Cette incroyable odyssée de l'or européen signifia la fin du système monétaire basé sur l'or qualifié par Keynes de « relique barbare ». En 1944, les accords de Bretton Woods créeront une nouvelle organisation monétaire internationale dans laquelle le dollar américain, désormais monnaie de réserve internationale, sera la seule monnaie indexée sur l'or.

53 Winston Churchill essaiera de récupérer les 254 tonnes d'or transférées d'Halifax à Fort-deFrance, lors de l'Opération Astérisk en septembre 1940.

54 Banque du Canada, Final report on wartime gold transactions released, November 27, 1997, https://www.bankofcanada.ca/1997/11/final-report-wartime-gold-transactions-released/ . 